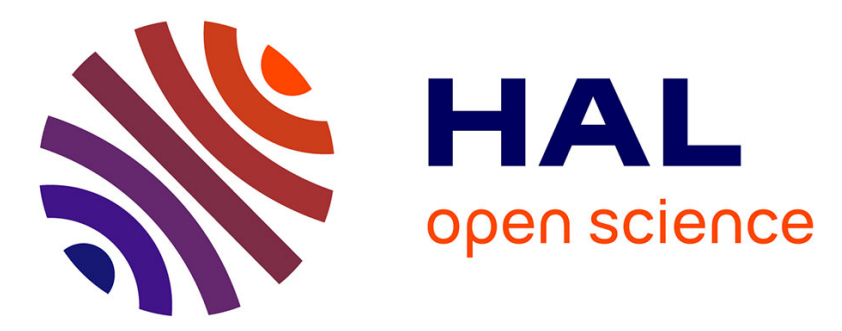

\title{
Dynamic Spatial Formation and Distribution of Intrinsically Disordered Protein Droplets in Macromolecularly Crowded Protocells
}

Hang Zhao, Vusala Ibrahimova, Elisabeth Garanger, Sébastien

Lecommandoux

\section{To cite this version:}

Hang Zhao, Vusala Ibrahimova, Elisabeth Garanger, Sébastien Lecommandoux. Dynamic Spatial Formation and Distribution of Intrinsically Disordered Protein Droplets in Macromolecularly Crowded Protocells. Angewandte Chemie International Edition, 2020, 59 (27), pp.11028-11036. 10.1002/anie.202001868 . hal-02519909

\section{HAL Id: hal-02519909 \\ https://hal.science/hal-02519909}

Submitted on 6 Apr 2020

HAL is a multi-disciplinary open access archive for the deposit and dissemination of scientific research documents, whether they are published or not. The documents may come from teaching and research institutions in France or abroad, or from public or private research centers.
L'archive ouverte pluridisciplinaire HAL, est destinée au dépôt et à la diffusion de documents scientifiques de niveau recherche, publiés ou non, émanant des établissements d'enseignement et de recherche français ou étrangers, des laboratoires publics ou privés. 
Dynamic Spatial Formation and Distribution of Intrinsically Disordered Protein Droplets in Macromolecularly Crowded Protocells

\author{
Hang Zhao, ${ }^{[a]}$ Vusala Ibrahimova, ${ }^{[a]}$ Elisabeth Garanger ${ }^{[a]}$ and Sébastien Lecommandoux ${ }^{*[a]}$
}

[a] Dr. H. Zhao, Dr. V. Ibrahimova, Dr. E. Garanger, Prof. Dr. S. Lecommandoux

Univ. Bordeaux, CNRS, Bordeaux INP, LCPO, UMR 5629, F-33600, Pessac, France

E-mail: sebastien.lecommandoux@enscbp.fr

Supporting information for this article is given via a link at the end of the document.

\begin{abstract}
Elastin-like polypeptides (ELPs) have been proposed as a simple model of intrinsically disordered proteins (IDPs) which can form membrane-less organelles via liquid-liquid phase separation (LLPS) in cellular milieu. Herein, fluorescently labeled ELP is studied in cytomimetic aqueous two-phase system (ATPS). Droplet-based protocells are obtained in a microfluidic system, allowing for confinement, temperature changes and statistical analysis. The spatial organization of ELP is observed in such binary ATPS macrocrowders. In addition, owing to switch of conformational states, dynamic formation and distribution of ELP-rich droplets within the artificial cytoplasm is triggered by temperature. Three-dimensional structured proteins are concurrently encapsulated along with ELP in synthetic cells and distinct partitioning properties of these proteins and ELP in binary polymeric phases are observed. This underpinning discovery demonstrates that the ability of ELP to coacervate with temperature can be maintained inside intracellular mimetic medium, and the preferential distribution of ELP in macromolecular crowding.
\end{abstract}

\section{Introduction}

Compartmentalization of biocomponents is one of the essential and vital biological functions for the evolution of living systems. Cell, the basic unit of life, is certainly the most recognized biological compartment that shares this function known as cellularization, that exists in spatiotemporally concentrated and localized reactants or protect biomaterials from damaging environment. ${ }^{[1]}$ Inside cells, cellular materials are further compartmentalized into organelles, that are either membranebound vesicles or membrane-less structures, providing an organization of bioactivity. ${ }^{[2]}$ Membrane-less organelles have aroused enormous interest for their important role in many cellular processes, ${ }^{[3]}$ and significant efforts have been provided to identify the molecular principles underlying their formation. ${ }^{[4]}$ Remarkable progress has been made in field of complex coacervates based membrane-less compartments that are believed holding great promise for mimicking subcellular entities. ${ }^{[5]}$ Pioneering studies were especially carried out by Mann and Keating, who recently demonstrated that dynamic assembly/disassembly of membrane-less microdroplets based upon complex coacervation can be modulated in response to environmental stimuli, for example light or enzyme activity. ${ }^{[6]}$ Over the last decade, with compelling evidence accumulated, there has been a significant increasing awareness that nonmembrane subcellular structures in living cells are liquid-like assemblies that generally contain RNA and intrinsically disordered proteins (IDPs). It is now well admitted that they are formed by spontaneous liquid-liquid phase separation (LLPS), since Brangwynne and his colleagues discovered $P$ granules in embryo, ${ }^{[7]}$ heralding cell biology steps into a new phase. ${ }^{[8]}$ IDPs that are seen as one of the most important drivers for constructing such liquid-like bodies, ${ }^{[9]}$ have been estimated to occupy over $30 \%$ among proteins in eukaryotic cell. ${ }^{[10]}$ They are lacking the traditional ordered three-dimensional structures existing in conventional proteins, and many of them reveal highly repetitive and low complexity sequences. IDPs based on elastinlike polypeptide (ELP) sequences have been recently reported to undergo thermoresponsive phase transition and exhibit similar structures to phase separated IDP in membrane-less organelles. ${ }^{[11]}$

ELPs are synthetic biopolymers consisting of repetitive pentapeptide sequences Val-Pro-Gly-Xaa-Gly, (VPGXG), where the guest residue $(X)$ can be any amino acid with the exception of proline. ${ }^{[12]}$ The particular feature of ELPS is to exhibit lower critical solution temperature (LCST) phase behavior in water below their phase transition temperature $\left(T_{\mathrm{t}}\right)$ they are soluble, while above their $T_{\mathrm{t}}$ they undergo simple phase separation to form an ELP-rich coacervate, which is driven by an entropic process due to hydrophobic interactions between ELP chains. ${ }^{[12 b, c]}$ Due to these unique characteristics, ELPs have been reported as an extremely simple but relevant analogue of IDP. [13] Recently, a simple protocellular compartment was described by compartmentalizing a library of ELPS or ELP-RNAbinding domain inside water-in-oil emulsions to either study their ability to form multilayered orthogonal coacervates and variable size of hydrogels, or engineer membrane-less organelle of ELP. RNA condensates that inhibits translation. ${ }^{[14]}$ However, in those cases ELPs were mostly manipulated in simple aqueous medium, lowering the system complexity and limiting the understanding of myriad possibilities of how IDP operates in cell cytoplasm. The interior of natural cells is indeed a crowded milieu, where IDPs abundantly locate and operate with many other biomacromolecules. ${ }^{[15]}$ An aqueous two-phase system (ATPS) composed of two neutral polymers such as dextran and poly(ethylene glycol) (PEG) can be viewed as a simple model system to mimic this intracellular organization, and has been extensively studied in bulk or encapsulated inside droplets and vesicles. ${ }^{[16]}$ The effect of such macromolecular crowding on structures and functions of ordered proteins is rather well documented. However, much less is known about how the complexity of the cell interior might affect structural and functional behavior of IDPs that can themselves phase separate. In the present paper, we first demonstrate that in bulk phase ATPS emulsions, ELP shows preferential localization between binary polymeric phases. However, phase separation of ELP has not been observed efficiently when sample is heated above $T_{\mathrm{t}}$. Next, two types of microdroplet-based cell-like constructs are generated by microfluidics, in which synthetic cytoplasmic organization (ATPS) and fluorescently labelled ELP (artificial IDP) with/without well-defined natural proteins and enzymes namely bovine serum albumin (BSA), horseradish peroxidase (HRP) and concanavalin A (Con A), are simultaneously present. When temperature is set below $T_{\mathrm{t}}$, ELP exhibits similar pronounced partitioning preference between two polymeric phases in protocell constructs. Upon heating, ELP is able to respond to temperature change and phase separate, enriching at liquid-liquid interface of ATPS or internalizing into dispersed dextran phase. The process of coacervation and spatial distribution is completely reversible upon lowering the temperature below the $T_{\mathrm{t}}$ of ELP. To further increase complexity of artificial cytoplasm, proteins of BSA, HRP and Con A, are individually added to ATPS and ELP system. Through injection into oil phase at high temperature, single emulsions are formed holding a homogenous phase in the compartments. With temperature decreasing below ELP's transition temperature, temperature-regulated phase separation of ATPS can rapidly occur and ELP consequently enriches in one phase of dextran/PEG system. In addition, specific partitioning behaviors 
of each protein between dextran and PEG phases can be seen Similar to ELP behaviors at high or low temperature in previous case, dynamic formation/dissolution and re-localization of ELP coacervates can be clearly observed. Our findings based upon combination of ELP and artificial cytoplasmic system, especially when they are compartmentalized inside protocells, may allow for the building of a cell-mimicking system with a higher degree of hierarchical complexity.

\section{Results and Discussion}

Aqueous biphasic systems, namely mixtures of two neutral polymers or a polymer and a salt, can phase separate through segregation into two regions where one polymer is enriched in another component, driven by predominantly repulsive interactions between macromolecules. ${ }^{[17]}$ To mimic biomacromolecules crowded nature of cells' interior, ${ }^{[15]}$ a classical solute pair of dextran and PEG was used in the present work. Indeed, it has been reported that at certain molar masses and concentrations of each polymer, segregative phase separation can be initiated by temperature ${ }^{[17-18]}$ Considering that thermo-responsive ELP is incorporated with the ATPS, phase diagrams of dextran/PEG system at $10^{\circ} \mathrm{C}$ and $50^{\circ} \mathrm{C}$ (Figure $\mathrm{S} 1$, Supporting Information) were determined by cloud point titration method to identify threshold concentrations under which ATPS could demix, forming two immiscible aqueous phases. In this phase diagram, regions of two-phase and single-phase were segregated by coexistence curves, where phase separation is observed above the curve, while the ATPS solution exists as a homogeneous phase below the curve. ${ }^{[16 a]}$ Varying temperature from $10^{\circ} \mathrm{C}$ to $50^{\circ} \mathrm{C}$, the curve of phase diagram shifted towards higher concentration of PEG and dextran, leading to an increased compatibility of the system. To generate bulk phase ATPS water-in-water droplets, which are further used to investigate partitioning behavior of the ELP, various concentration ratios between dextran and PEG in the two-phase region were tested. In a typical experiment, the concentrations used for dextran and PEG were $4 \mathrm{wt} \%$ and $8 \mathrm{wt} \%$, respectively, allowing the formation of stable dextran-in-PEG droplets at both $10^{\circ} \mathrm{C}$ and $50^{\circ} \mathrm{C}$ (Figure S1, Supporting Information).

ELPs that serve as model IDPs for their temperature-sensitive phase behavior have been widely characterized and have been demonstrated to build liquid granules inside microdroplets. ${ }^{[14 a, b, 19]}$ We specifically focused this study on ELP containing periodically spaced methionine residues in the guest position, namely ELP $\left[M_{1} \bigvee_{3}-60\right]$ following established nomenclature (Figure S2, Supporting Information). ${ }^{[20]}$ This ELP was produced recombinantly in Esherichia coli bacteria and purified by inverse transition cycling (ITC) as described previously. ${ }^{[19,21]}$ In order to visualize preferential localization of artificial IDP inside dextran/PEG system, a BODIPY dye was selected to fluorescently label ELP[M $\left.\mathrm{V}_{3}-60\right]$ at the $N$-terminal end (Figure S3 and S4, Supporting Information). To examine purity of BODIPY labelled ELP, named BDP-ELP[M $\left.\mathrm{M}_{1}-60\right]$, SDS-PAGE electrophoresis analysis was conducted on the ELP after purification by ITC. This analysis evidenced that the fluorescent ELP product is free of excess dye molecules (Figure S5, see the Supporting Information for full experimental detail). We then evaluated the transition temperature $\left(T_{t}\right)$ of the resulting BODIPY-labeled ELP at 0.25 and $0.125 \mathrm{mg} \mathrm{mL}^{-1}$ in each polymer phase, respectively (dextran $4 \mathrm{wt} \%$ or PEG $8 \mathrm{wt} \%$ ) (See more details in the Supporting Information). At both concentrations of 0.25 and $0.125 \mathrm{mg} \mathrm{mL}^{-1}$ in dextran solution, BDP-ELP $\left[\mathrm{M}_{1} \mathrm{~V}_{3}-60\right]$ exhibited phase transition around $30^{\circ} \mathrm{C}$ (Figure S6, Supporting Information), confirming that fluorescently tagged ELP can experience thermally induced phase transition in between 10 to $50^{\circ} \mathrm{C}$ in only dextran and PEG aqueous phases of the ATPS system. However, we have noticed that transition temperatures of ELP in macromolecular crowding environment shift to lower temperature, which can be attributed to the increased hydrophobicity of ELP when mixed with PEG or dextran, as a result of the interaction between macromolecules and water molecules that leads to the reduction of swelling of polypeptide chains.

To determine the effect of microcompartmentalization induced by phase separation of ATPS on the distribution of ELP and its ability to form coacervates, BDP-ELP $\left[\mathrm{M}_{1} \mathrm{~V}_{3}-60\right]$ in APTS was analyzed by fluorescent confocal microscopy. As temperature is a crucial parameter to control over ELP phase state, we compared behaviors of the ELP when temperature is above and below $T_{\mathrm{t}}$. We first tested BDP-ELP[M $\left.\mathrm{M}_{1} \mathrm{~V}_{3}-60\right]\left(0.25 \mathrm{mg} \mathrm{mL}^{-1}\right)$ within dextran/PEG mixture through vortex mixing, generating dextran-in-PEG bulk phase emulsions. Initially the bulk emulsions were imaged at $10^{\circ} \mathrm{C}$, below ELP transition temperature (Figure $1 \mathrm{a}$ ). Dextran-in-PEG droplets were well fabricated, highlighted by tracking fluorescein labelled dextran. ELP showed markedly uneven spatial distribution between dextran/PEG phases, with no obvious accumulation in the dextran droplets, but significant BODIPY signal was detected in the continuous PEG region, indicating BDP-ELP[M $\left.\mathrm{V}_{3}-60\right]$ can partition towards PEG fraction in a highly selective manner. We reasoned that this strong partitioning could be attributed to the molecular structure of PEG which differs from that of dextran. ${ }^{[22]}$ Indeed, PEG is a highly hydrophilic polymer that contains highly coil-like flexible chain and ELP is in fully hydrated polymer chainlike conformation at $10^{\circ} \mathrm{C}$ (below the $T_{\mathrm{t}}$ ), resulting in the preferential distribution of the ELP into PEG phase (Figure 1 a2), as compared to dextran that is stiffer and less hydrated at this temperature. To assess the influence of thermally induced phase transition of ELP on its distribution within bulk ATPS emulsions, we then monitored the BODIPY fluorescence signal upon heating the sample above its $T_{\mathrm{t}}$ up to $50^{\circ} \mathrm{C}$. At high temperature, dextran droplets structurally remained stable (Figure $1 \mathrm{~b} 1$ ). As a consequence of the conformational change from coiled chain structure to coacervate form at the temperature higher than $T_{\mathrm{t}}$, ELP is expected to phase separate in the PEG-rich phase. A spatially inhomogeneous organization of ELP and decrease of fluorescence occurred at $50^{\circ} \mathrm{C}$, as a result of nanoscale ELP coacervates that were formed during the increase of temperature in the imaging chamber (diameter 9 $\mathrm{mm}$; thickness $0.12 \mathrm{~mm}$ ); however nano-sized coacervates would be fairly diluted in PEG environment consequently leading to undetectable coacervation of ELP based upon fluorescence imaging (Figure $1 \mathrm{~b} 2$ ). We further decreased the temperature back to $10^{\circ} \mathrm{C}$, and the same initial fluorescence levels were observed (Figure $1 \mathrm{c}$ ), attesting the reversibility of the process. From this first series of experiments, one can then conclude that our artificial IDP based on BDP-ELP[M $\left.\mathrm{V}_{3}-60\right]$ specifically localizes in the PEG phase of the APTS independent on the applied temperature.

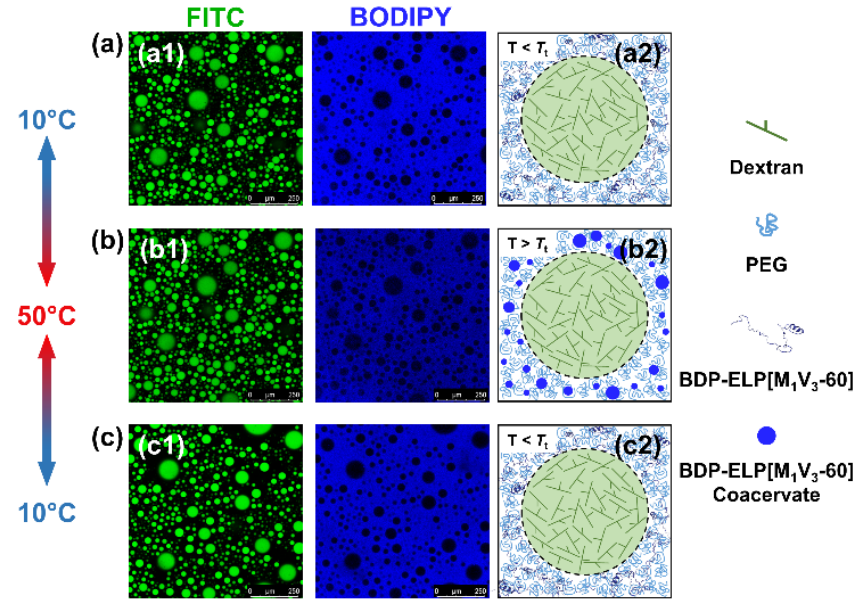


Figure 1. Distribution of BDP-ELP $\left[\mathrm{M}_{1} \mathrm{~V}_{3}-60\right]$ in the bulk phase dextran/PEG emulsion system. (a.b.c) Confocal images and schemes (not to scale) of BDPELP $\left[\mathrm{M}_{1} \mathrm{~V}_{3}-60\right]$ within dextran-in-PEG droplets at 10,50 and $10^{\circ} \mathrm{C}$ respectively. (a) $10^{\circ} \mathrm{C}$. (b) $50^{\circ} \mathrm{C}$. (c) $10^{\circ} \mathrm{C}$

Despite size of bulk phase dextran-in-PEG emulsions lie in microscale, PEG-rich region is a macroscopic phase in which as-formed ELP coacervates might be at relatively low local concentrations, resulting in difficulties of visualizing phase separation efficiently. Water-in-oil emulsions constructed by droplet-based microfluidics are simple yet powerful entities to encapsulate multiple complex solutions towards synthesis of artificial cells. We then developed a microfluidic strategy to generate microdroplets in which nanoliter cytomimetic system and the IDP analogue were enclosed simultaneously. To emulsify two polymer phases of ATPS into single droplets surrounded by an oil continuous phase, we used a capillarybased microfluidic device. The device was built from a theta $(\theta)$ shape injection capillary with two separate channels that is tapered to a narrow tip and slightly inserted in a second cylindrical capillary which is used as collection channel for produced microdroplets (Figure 2 a). As demonstrated in previous bulk ATPS emulsions experiments, strikingly selective partition of ELP to PEG-rich phase occurred at both temperatures above and below $T_{\mathrm{t}}$. To examine partitioning ability of ELP, $0.5 \mathrm{mg} \mathrm{mL}^{-1}$ fluorescent BDP-ELP $\left[\mathrm{M}_{1} \mathrm{~V}_{3}-60\right]$ was mixed with $8 \mathrm{wt} \%$ of dextran (with $0.2 \mathrm{mg} \mathrm{mL}^{-1}$ FITC-dextran) in one stream and $16 \mathrm{wt} \%$ of PEG was flowed as the second solution through another channel. An oil phase (TEGOSOFT $\mathrm{DEC} /$ mineral oil) with a non-ionic surfactant (ABIL EM 90) was pumped into the device through the interstice between cylindrical and square capillaries to merge and pinch off two aqueous flows at the aperture forming water-in-oil microdroplets (Figure 2 b). Syringe pumps were utilized to introduce two aqueous streams into the device at the same flow rate. Therefore a volume ratio of 1:1 between the dextran and PEG phase could be precisely achieved, making sure that concentrations of solutes in as-prepared microdroplets were exactly the same as those in previously prepared bulk phase emulsions.

By fabricating droplets via microfluidics, monodispersed single emulsions and interior phase-separate dextran droplets were produced with mean diameters of $195 \mu \mathrm{m}$ and $113 \mu \mathrm{m}$ respectively (Figure $2 \mathrm{c}, \mathrm{d}$ ), indicating every single microdroplet harbors identical contents of dextran/PEG system as well as the fluorescent ELP. As-formed droplets were collected using continuous oil solution and imaged immediately at $10^{\circ} \mathrm{C}$. We likewise observed that BDP-ELP $\left[\mathrm{M}_{1} \mathrm{~V}_{3}-60\right]$ rapidly partitioned towards PEG-rich phase from dextran solution, which is in good agreement with our previous observations in bulk ATPS emulsions (Figure $2 \mathrm{c}$ ). After the incubation at $10^{\circ} \mathrm{C}$ for 15 minutes, ELP distribution was in equilibrium and its spatial organization inside cell-like chassis was analyzed in detail by confocal microscopy. According to the image (Figure 2 e), fluorescence intensities of both ELP and FITC-dextran can be acquired by line intensity profile (measured droplets $n=30$ ), allowing quantitative determination of distribution of each fluorescent product within protocells. In the corresponding intensity profile (Figure $2 \mathrm{f}$ ) it is clearly seen that a significant amount of ELP appeared in PEG-rich environment and the fluorescence signal emitted from ELP dramatically dropped $90 \%$ in the dextran-rich lumen, where in contrast with FITC-dextran signal remained steadily. (a)

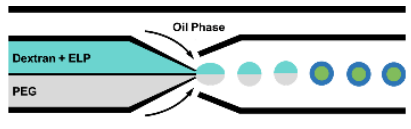

(c)
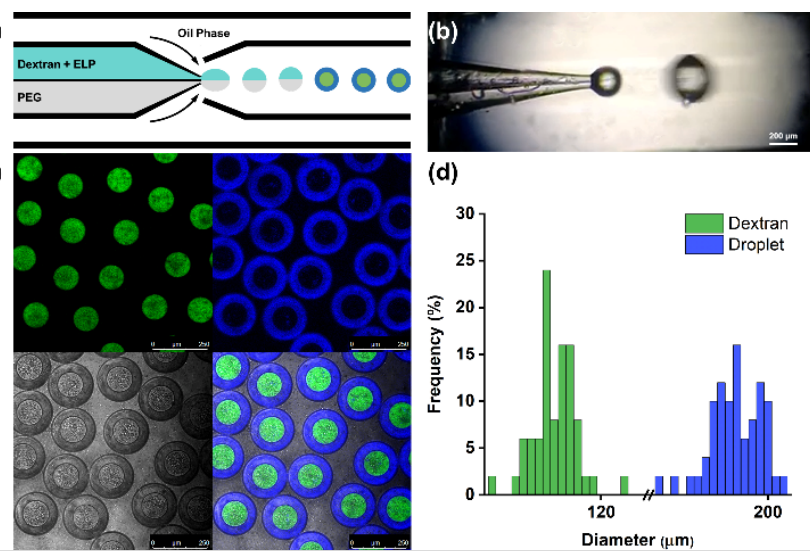

(d)

(e)
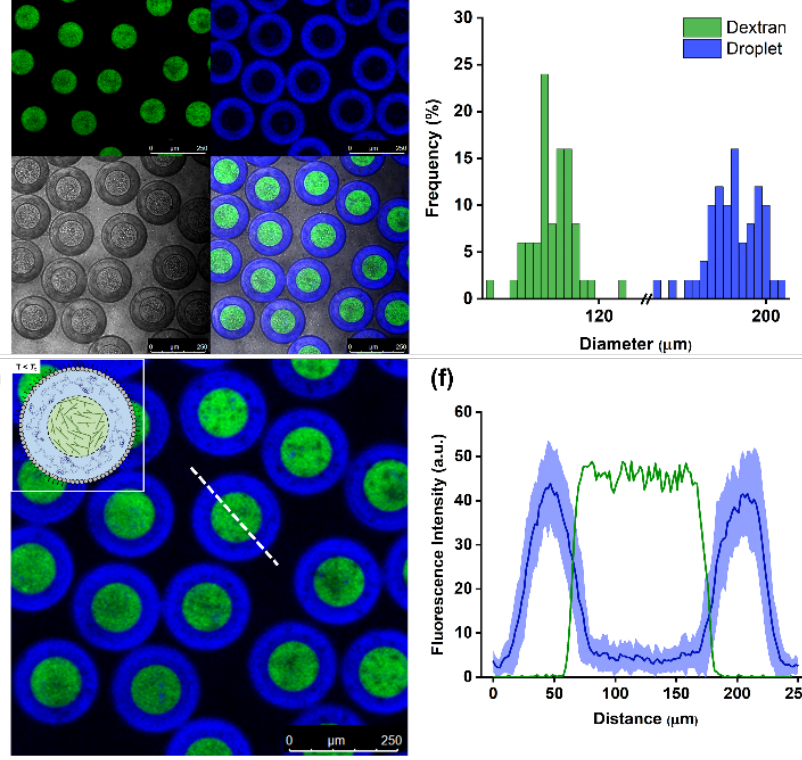

(f)

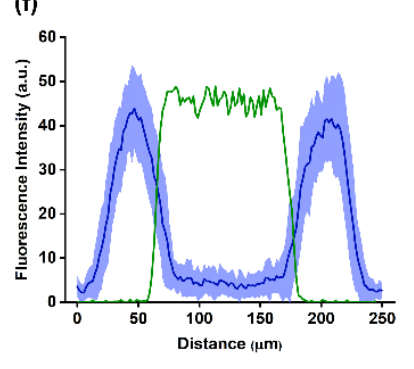

Figure 2. Encapsulation of BODIPY labelled ELP inside protocellular compartments via paired emulsions. (a) Illustration and (b) optical image of formation of paired emulsions by microfluidics; here, the upper phase is dextran and ELP while the lower phase is PEG. (c) Confocal images of asprepared microdroplets where green indicates dextran phase labelled by FITC-dextran; blue represents BODIPY tagged ELP that concentrates in the PEG phase; bright field and merged channel. (d) Size distribution of emulsions formed via microfluidics. Green bars indicate size of dextran lumen; blue is for droplet overall diameter. (e) Confocal image of microdroplets at $10^{\circ} \mathrm{C}$. Inset in (e) shows scheme of physical states of components inside droplets. (f) Fluorescence intensity profile shows distribution of dextran and ELP below $T_{\mathrm{t}}$, for example corresponded to white dash line in (e). Droplet number for fluorescence intensity profile $n=30$. Plots show the average values, with the shaded region indicating standard deviation.

To determine how the confinement may impact on thermalresponsive phase separation of the ELP, the droplets were heated well above the $T_{\mathrm{t}}\left(50^{\circ} \mathrm{C}\right)$, and image acquisition was started 15 minutes equilibrium to guarantee a uniform temperature within imaging petri-dish. Interestingly, in such cellmimicking compartments, phase transition of the ELP was thermally triggered and resulted in the formation of clearly visible coacervates, whose dynamics could be readily followed. As shown in Figure $3 \mathrm{a}$, at $50^{\circ} \mathrm{C}$, dextran lumen was thoroughly encircled by phase separated ELP entities, denoting these puncta bodies condensed from PEG-rich and progressively redistributed mostly at the interface of dextran/PEG phases. Having ELP conformation changed into protein-rich assemblies at a temperature well above the $T_{\mathrm{t}}$, within dextran/PEG system, the PEG phase largely excludes these coacervates since it is occupied by flexible chains and therefore cannot provide ideal environment for ELP-rich droplets. On the contrary, nanosized void space, existing in the dextran phase, is driving these ELP droplets to move at the interface and even for some part internalize into the dextran lumen (Figure $3 \mathrm{~b}$ ). Consistently, in the three-dimensional surface plot (Figure $3 \mathrm{c}$ ), the fluorescence signals of BDP-ELP $\left[\mathrm{M}_{1} \mathrm{~V}_{3}-60\right]$ at dextran/PEG interface and some spots in dextran lumen were remarkably stronger than the surrounding PEG phase. 
(a)
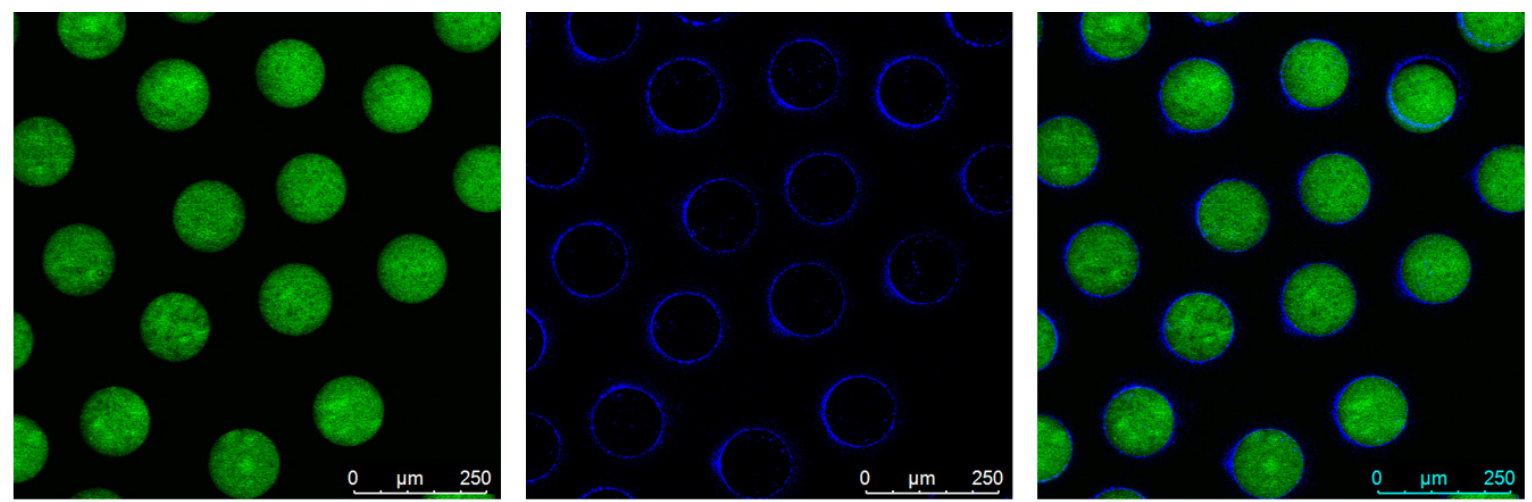

(b)
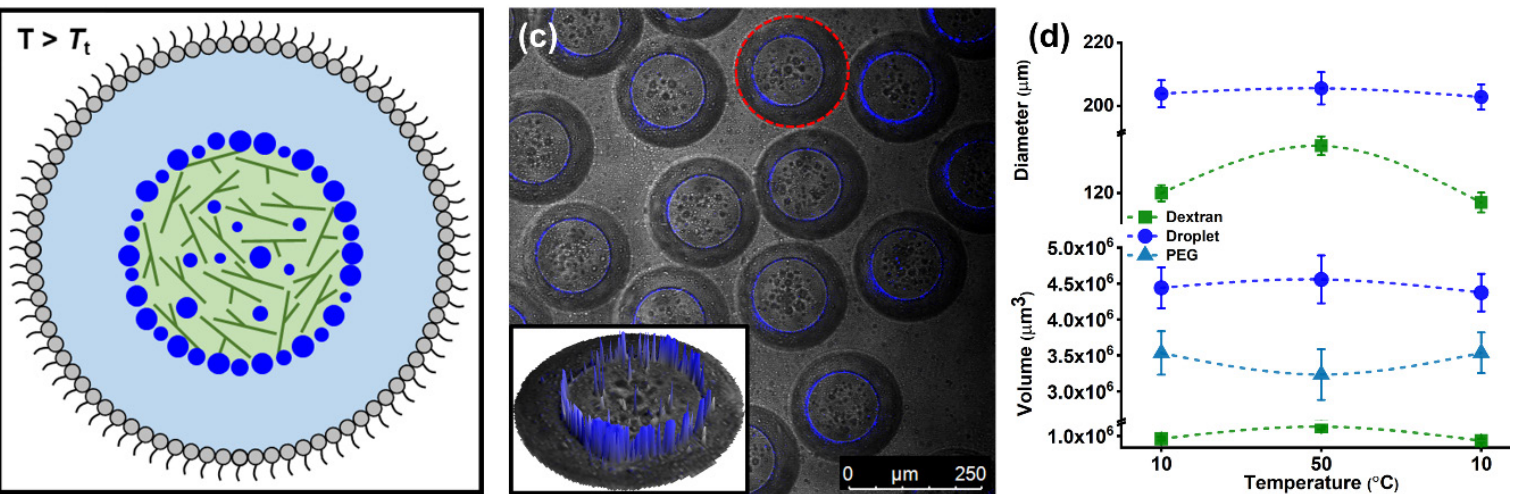

(e)
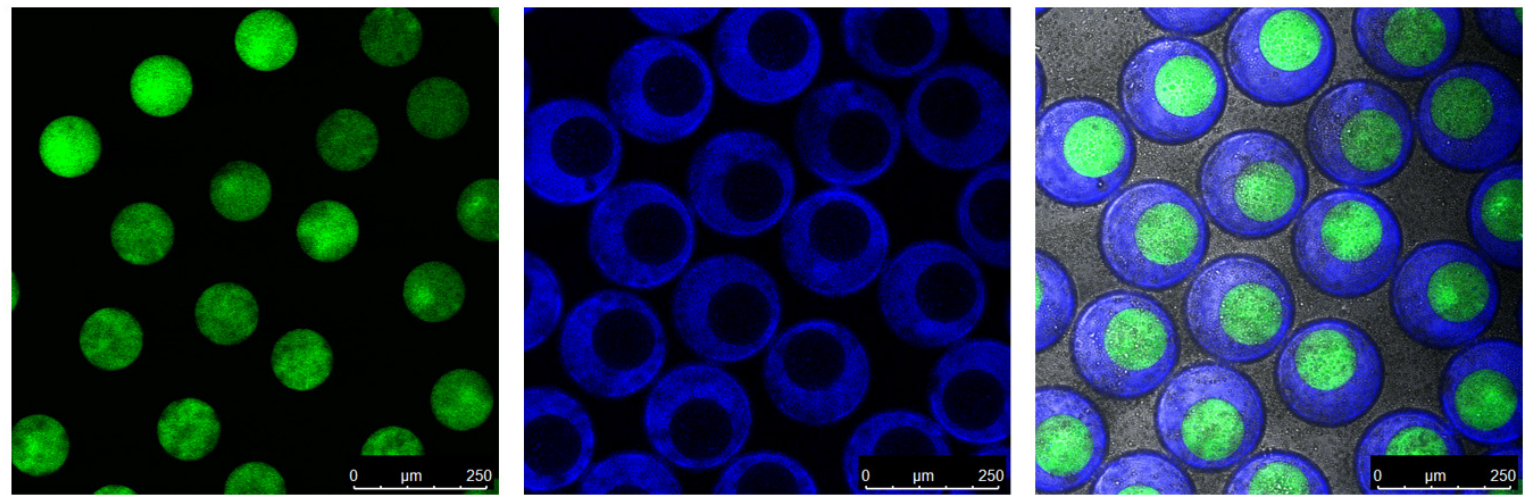

Figure 3. Coacervation and dynamic distribution of BDP-ELP $\left[M_{1} \vee_{3}-60\right]$ in microdroplets at $50^{\circ} \mathrm{C}$ and $10^{\circ} \mathrm{C}$. (a) At $50^{\circ} \mathrm{C}$, ELP coacervates were formed and directed to the dextran/PEG interface. Green indicates FITC-dextran and blue is for BDP-labeled ELP. (b) Schematic illustration of ELP behavior in compartments when $T$ is above $T_{\mathrm{t}}$. (c) Merged channel of bright field and blue channel of microdroplets imaged at $50^{\circ} \mathrm{C}$; (inset) $3 \mathrm{D}$ surface plot shows fluorescence intensity coming from ELP coacervates in the region of interest indicated by red dash circle in (c). (d) Upper: size variation of dextran lumen and microdroplets against temperature changes; bottom: volume variation of dextran lumen, PEG phase and microdroplets in response to temperature changes (number of droplets measured $n=30$ ). (e) Rehydration and relocation of the ELP when temperature is cooled down to $10^{\circ} \mathrm{C}$, below $T_{\mathrm{t}}$. 
Therefore, observations in these microdroplets differed from bulk phase emulsions, both in terms of the formation of ELP coacervates and their spatial re-organization. We reasoned the different behaviors stem from two aspects: (i) the volume in a microdroplet is around 4.38 nanoliter, as compared to the volume of an imaging spacer that is 7634 nanoliter, meaning that in protocellular compartments the local concentration of condensed coacervates would be higher. This difference in local concentration of ELP coacervates allowed these puncta to readily fuse into larger size and spatially arrange towards the interface and dextran core; (ii) interestingly, we found during the incubation at $50^{\circ} \mathrm{C}$, the PEG phase dehydrated as attested by its decreased average volume while the dextran phase enriched in water and increased its overall volume (Figure $3 \mathrm{~d}$ ). This consequently led to a slight increase of ELP concentration in PEG phase, which may promote its coacervation and relocalization. Taken together, the strategy of microcompartmentalization enables a particularly efficient phase separation and associated dynamic distribution of ELP-rich assemblies to occur, offering increased feasibility of using droplet-based protocells to investigate IDPs. Furthermore, to demonstrate the reversibility of this dynamic assembly/disassembly phenomenon of such protein-rich droplets and their spatial organization, the temperature was lowered back to $10^{\circ} \mathrm{C}$. The ELP coacervates readily rehydrated and re-dispersed, leading back to the same initial repartition into PEG-rich phase (Figure $3 \mathrm{e}$ ). To further confirm this specific behavior of our artificial IDP in cellular mimetic system, we reduced ELP concentration to $0.125 \mathrm{mg} \mathrm{mL}^{-1}$ and observed the exact same phenomenon (Figure S7, Supporting Information). Based upon these observations, the compartmentalized temperature responsive ELP can not only toggle between homogenous phase and phase-separated coacervate, but also can reversibly and dynamically move between PEG-rich phase and dextran-PEG interface. Such mimic of the dynamics of reversible assembly of protein coacervates in polymer-based cytomimetic system can provide enlightened understanding of coacervation of protein clusters within subcellular compartments. ${ }^{[23]}$

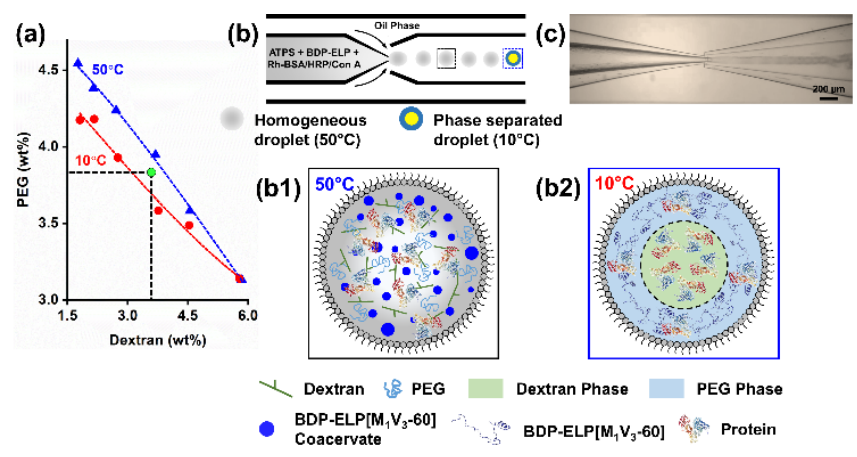

Figure 4. (a) Phase diagram in which a green circle marks the composition used in this experiment (3.6 wt\% dextran and $3.8 \mathrm{wt} \% \mathrm{PEG}$ ). (b) Illustration of microdroplets formed by single emulsion microfluidic device. Grey-colored droplet indicates its homogeneous form at $50^{\circ} \mathrm{C}$; Yellow-blue core-shell drople represents droplet with phase separation of ATPS, ELP and protein. (b1) Scheme of a microdroplet prepared at $50^{\circ} \mathrm{C}$ prior to phase separation showing homogeneous spatial distribution of all components; (b2) Scheme of a microdroplet at $10^{\circ} \mathrm{C}$ in which ATPS and protein phase separate, with ELP largely enriched in PEG phase. (c) Optical image of generation of water-in-oil single emulsions at $50^{\circ} \mathrm{C}$

In living cells, membrane-less subcellular structures are ubiquitous, and enable spatiotemporal control of biological reactions. These membrane-less organelles are liquid-like architecture, that form via protein phase separation in the cytoplasm. ${ }^{[24]}$ Having accomplished dynamic modulation of assembly/disassembly of phase-separated IDP-rich droplets and spatial regulation of their localization inside cellular compartments with artificial cytoplasm encapsulated, we further explored cell-mimicking potential of our macromolecularly crowded protocells. To this end, we extended the complexity of the cytomimetic system, three types of well-structured and functional proteins: BSA, HRP and Con A were individually introduced to a single-phase ATPS together with BDP$E L P\left[M_{1} V_{3}-60\right]$. All these proteins were labelled with rhodamine fluorophore allowing to spectrally visualize both protein and ELP in protocells. We started with a solution mixture that is preheated at $50^{\circ} \mathrm{C}$ including a single-phase ATPS, ELP and single protein. By changing temperature below or above ELP $T_{\mathrm{t}}$, both processes of partitioning of ELP and proteins along with phase separation of one-phase dextran/PEG system were monitored, and dynamic coacervation of ELP was also investigated. In this instance, to firstly reach a single-phase ATPS, a concentration ratio of $3.6 \mathrm{wt} \%$ dextran and $3.8 \mathrm{wt} \%$ PEG was used upon the phase diagram. The temperature-dependent phase separation property of ATPS was confirmed in bulk solution (Figure 4 a and Figure S8, Supporting Information). Microfluidic device was developed by replacing the theta $(\theta)$ shape capillary with a single channel cylindrical capillary to produce water-in-oil single emulsions at high-throughput (Figure $4 \mathrm{~b}, \mathrm{c}$ ). Typically, a solution of ATPS of $3.6 \mathrm{wt} \%$ dextran (0.1 $\mathrm{mg} \mathrm{mL}^{-1}$ FITC-dextran) and 3.8 wt $\%$ PEG, and $0.25 \mathrm{mg} \mathrm{mL}^{-1}$ of both ELP and protein was premixed at $50^{\circ} \mathrm{C}$ and used as dispersed phase. The same oil phase (TEGOSOFT DEC/mineral oil) was used as continuous phase. To tune the generation of emulsion droplets in the dripping regime, typical flow rates for aqueous and oil phase were 500 and $5000 \mu \mathrm{L} \mathrm{h}^{-1}$, respectively. It was important that during the formation process, the aqueous phase was kept at $50^{\circ} \mathrm{C}$, rendering a homogenous cyan-colored phase inside single emulsions, attesting that green fluorescent dextran tracer, rhodamine-protein (red) and BODIPY-ELP coacervates (blue) were uniformly localized within protocells (Figure $4 \mathrm{~b} 1$ ). Upon cooling to $10^{\circ} \mathrm{C}$ below ELP $T_{\mathrm{t}}$, spontaneous phase demixing of dextran/PEG system drives rehydrated ELP and FITC-dextran into PEG and dextran regions separately, while the distribution of protein depends on its nature and interactions with each $\begin{array}{lllll}\text { polymeric } & \text { phase } & \text { (Figure } & 4 & \text { b2) }\end{array}$ 


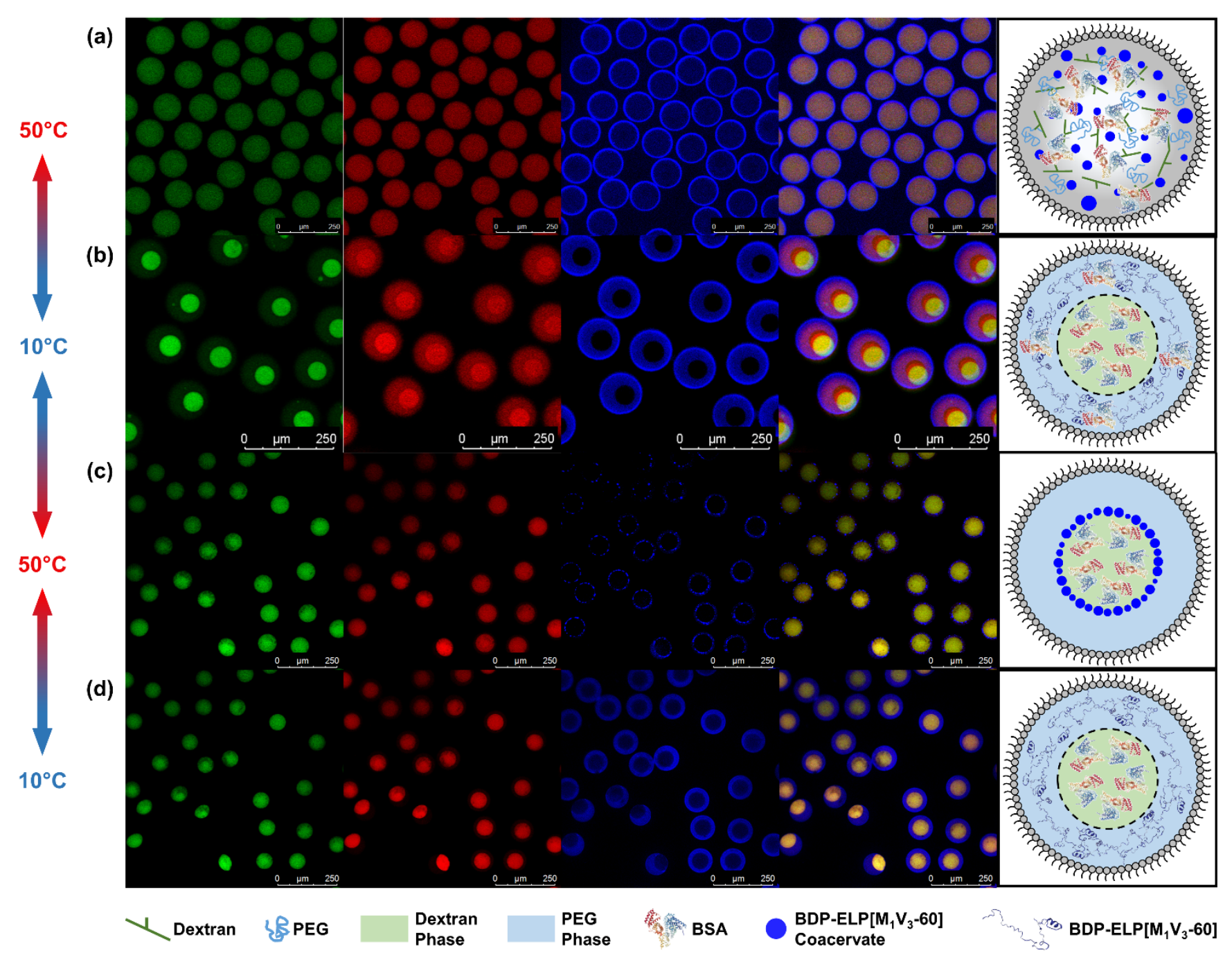

Figure 5. Representative confocal images of Rh-BSA, BDP-ELP $\left[M_{1} V_{3}-60\right]$ and ATPS within microdroplets at different temperatures showing thermally induced dynamic organization and motion of the ELP-rich droplets and spatial organization of protein and ELP. (a) Microdroplets are initially imaged at $50^{\circ} \mathrm{C}$. (b) $10^{\circ} \mathrm{C}$. (c) $50^{\circ} \mathrm{C}$ and $(\mathrm{d}) 10^{\circ} \mathrm{C}$.

First, we tested Rh-BSA using the aforementioned approach. After production and collection of microdroplets off the microfluidic device, single emulsions were imaged instantly at $50^{\circ} \mathrm{C}$. Figure 5 a shows that inside monodispersed microdroplets, fluorescence signals for FITC-dextran, rhodamine and BODIPY were uniform, indicating that a homogenous phase was successfully created. To activate the phase separation, temperature was set down to $10^{\circ} \mathrm{C}$ at which dextran and $P E G$ will demix from homogenous phase creating a two-phase system. With decreasing temperature, the onset of phase transition was initially captured around $30^{\circ} \mathrm{C}$. Once temperature reached $10^{\circ} \mathrm{C}$, emulsions were subsequently maintained for 15 minutes. In this period, the dynamics of dextran condensed from surrounding ATPS was continuously monitored showing that small, discrete dextran droplets appeared and fused with one another, enlarging in size and eventually forming a dextran lumen (Video S1, Supporting Information). After reaching equilibrium at $10^{\circ} \mathrm{C}$, confocal images of these emulsions obviously exhibited that respective segregation of each components: Rh-BSA particularly enriched in dextran phase as native BSA molecules readily partition into dextran-rich phase whilst ELP mostly accumulated within PEG shell (Figure $5 \mathrm{~b}$ ). The observations revealed that ATPS for a given concentration ratio of dextran/PEG phases is strongly capable of selectively distributing substances between the two phases, for instances artificial IDP and specific three-dimensionally structures proteins. We expanded our experiment to evaluate behaviors and distribution of Rh-BSA and BODIPY-labeled ELP against raising temperature back to $50^{\circ} \mathrm{C}$ and examined the ability of ATPS to re-mix into a single phase. Interestingly, we found that dextran/PEG system retained its phase-separated form at high temperature resulting in BSA also maintaining the localization in dextran-rich phase confirmed by the colocalization of FITC and rhodamine fluorophores inside dextran lumen (Figure $5 \mathrm{c}$ ). Similar to what we observed in paired emulsions, ELP at $50^{\circ} \mathrm{C}$ phase separated forming coacervates that spatially distributed at the dextran/PEG interface. The formation of such a "coacervate membrane" may prevent the reorganization of ATPS back into a single phase by lowering the surface tension at the dextran/PEG interface (Figure $5 \mathrm{c}$ ), as polyelectrolytes and liposomes can do. ${ }^{[25]}$ Consequently, the associated dynamic distribution of the protein is inhibited, retaining its preferential localization in the dextran/PEG system. Upon lowering the temperature to $10^{\circ} \mathrm{C}$, striking phase transition only occurred to ELP that was rehydrated and preferentially localized in PEG-rich phase, while 
BSA was still preferentially localized in dextran lumen (Figure $5 d)$.

To show the flexibility and versatility of our cell-mimicking system, two other proteins were used as models to be incorporated in the artificial cytoplasm. One is HRP (Figure 6 ad), an enzyme widely used in biochemistry application, and the other is Con A (Figure $6 \mathrm{e}-\mathrm{h}$ ), a protein (lectin) that specifically binds to carbohydrate. These two proteins were also individually introduced to dextran/PEG/ELP system in the same manner by mixing at $50^{\circ} \mathrm{C}$ prior to being loaded into the microfluidic device to form single emulsions. Immediately after production, microdroplets contain Rh-HRP or Rh-Con A were imaged and analyzed at $50^{\circ} \mathrm{C}$. For protein of HRP, all fluorescently tagged substances were homogenously dispersed within protocellular system (Figure 6 a), in contrast to the case of Con A in which dextran was slightly driven separately from the single-phase ATPS system (Figure $6 \mathrm{e}$ ). We noted that Rh-Con A was rapidly and efficiently enriched inside dextran-rich phase thanks to its affinity nature of strong binding to carbohydrates, like dextran (Figure $6 \mathrm{e}$ ). ${ }^{[26]}$ With decreasing temperature below ELP transition temperature, for both HRP and Con A conditions, gradual demixing of dextran and PEG led to the simultaneously significant enrichment of ELP into PEG phase (Figure $6 \mathrm{~b}, f$ and Video S2, Supporting Information). With regard to distribution of HRP and Con A, no substantial difference was observed indicating HRP remained the homogeneity within ATPS; but Con
A continuously bound to dextran phase (Video S2, Supporting Information). To validate the temperature regulated assembly of ELP coacervates, both samples were heated to $50^{\circ} \mathrm{C}$ (Figure 6 $\mathrm{C}, \mathrm{g})$. We observed that regardless of the types of proteins that were co-encapsulated, ELP always efficiently formed coacervates that were preferentially directed towards the dextran/PEG interface (Figure $6 \mathrm{c}, \mathrm{g}$ ), in agreement with previous observations. In Figure $6 \mathrm{~g}$, we noticed that Con A aggregated inside lumen leading to an increase of fluorescence intensity, which could be explained by the denaturation of Con A due to the incubation at high temperature for 15 minutes. Upon cooling samples to $10^{\circ} \mathrm{C}$, the rapid dissolution of those ELP-rich droplets and re-localization of chain structured ELP into PEG phase were imaged as usual (Figure $6 \mathrm{~d}, \mathrm{~h}$ ). It is worth noting, unlike BSA and Con $A$ that preferentially accumulate in dextran-rich phase HRP did not strongly favor either phase, which is in agreement with reported previously results. ${ }^{[27]}$ In this way, we demonstrated that our macromolecularly crowded protocells are endowed with the ability of selectively separating proteins with/without welldefined structures via phase separation of binary polymer phases. Significantly, in complex synthetic cytoplasm, the striking temperature-mediated behavior of ELP is highly reproducible and fully reversible, providing an elegant starting point for research about dynamic coacervation within artificial cytoplasm. 


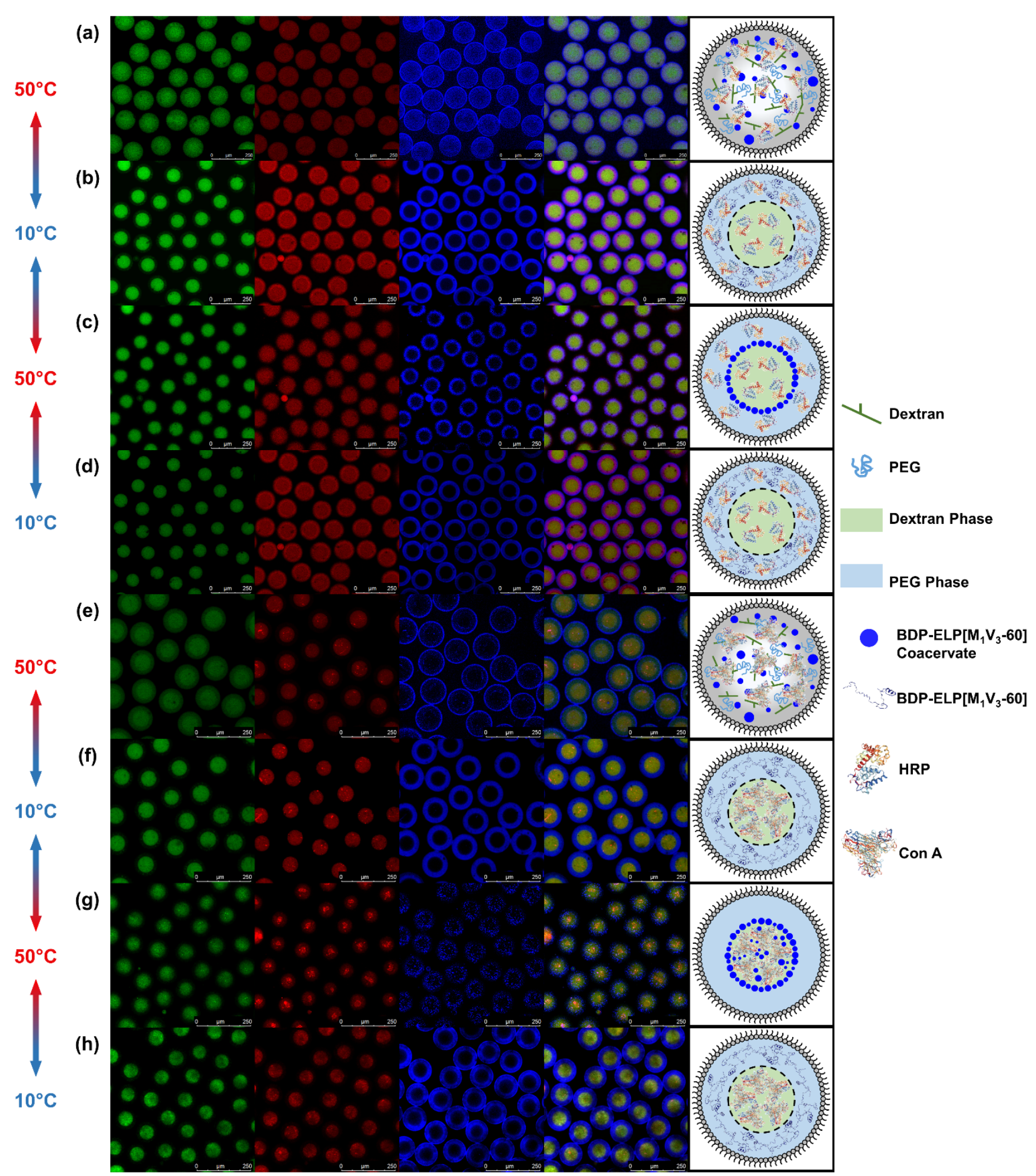

Figure 6. Representative confocal images of Rh-HRP, ELP with ATPS (a-d) and Rh-Con A, ELP with dextran/PEG system (e-h) within cell-like compartments at different temperatures showing thermal dynamics of the ELP coacervates and spatial organization of HRP, Con A and ELP. (a.e) Microdroplets are initially imaged at $50^{\circ} \mathrm{C}$. (b.f) $10^{\circ} \mathrm{C}$. (c.g) $50^{\circ} \mathrm{C}$ and (d.h) $10^{\circ} \mathrm{C}$. 


\section{Conclusion}

We have presented two compartmentalization strategies, namely bulk phase emulsion and cell-like compartment, to investigate the ability of phase separation and spatial distribution of an artificial IDP in a biomacromolecules crowded environment that mimics cell cytoplasm. The temperature-mediated dynamic and spatial control of coacervation of ELP in such cytomimetic compartment represented an advance towards synthetic cells by uniting IDP, ATPS, LLPS and compartmentalization. The distinct distribution property of ELP as a function of temperature allowed us to deepen insights into possible localization of other IDPs in the complex cellular milieu. Particularly, this work reported a robust approach to create protocellular systems with high-order interior complexity, which would pave the way on predicting distribution and phase-separating behavior of IDPs in macromolecularly crowded synthetic cells.

\section{Acknowledgements}

We wish to thank Bertrand Garbay and Guillaume Goudounet for gene design, cloning and production of the ELP, Katell Bathany for mass spectrometry analysis and Camille Legros for synthesis of Rh-BSA and Rh-HRP. We also wish to thank Evonik Industries for kindly providing products of TEGOSOFT DEC and ABIL EM 90. Euronanomed-ANR "Tempeat" project and IdEx Bordeaux are also gratefully acknowledged.

Keywords: aqueous two-phase system • elastin-like polypeptide - interfaces $\cdot$ phase transitions $\bullet$ synthetic protocells

[1] J. C. Holthuis, C. Ungermann, Biol. Chem. 2013, 394, 151-161.

[2] M. W. Gray, Trends Genet. 1989, 5, 294-299.

[3] a) G. Boulay, G. J. Sandoval, N. Riggi, S. Iyer, R. Buisson, B. Naigles, M. E. Awad, S. Rengarajan, A. Volorio, M. J. McBride, Cell 2017, 171 163-178; b) P. Li, S. Banjade, H.-C. Cheng, S. Kim, B. Chen, L. Guo, M. Llaguno, J. V. Hollingsworth, D. S. King, S. F. Banani, P. S. Russo, Q. X. Jiang, B. T. Nixon, M. K. Rosen, Nature 2012, 483, 336-340; c) J. R. Wheeler, T. Matheny, S. Jain, R. Abrisch, R. Parker, Elife 2016, 5 e18413-e18438; d) X. Su, J. A. Ditlev, E. Hui, W. Xing, S. Banjade, J. Okrut, D. S. King, J. Taunton, M. K. Rosen, R. D. Vale, Science 2016, 352, 595-599.

[4] Z. Feng, X. Chen, X. Wu, M. Zhang, J. Biol. Chem. 2019, 294, 1482314835.

[5] N. Martin, ChemBioChem 2019, 20, 2553-2568.

[6] a) W. M. Aumiller, C. D. Keating, Nat. Chem. 2016, 8, 129-137; b) N. Martin, L.-F. Tian, D. Spencer, A. Coutable-Pennarun, J. L. R. Martin, L.-F. Tian, D. Spencer, A. Coutable-Pennarun, J. L. R.
Anderson, S. Mann, Angew. Chem. Int. Ed. 2019, 58, 14594-14598; Angew. Chem. 2019, 131, 14736-14740; c) A. T. Rowland, D. N. Cacace, N. Pulati, M. L. Gulley, C. D. Keating, Chem. Mater. 2019, 31 10243-10255.

[7] C. P. Brangwynne, C. R. Eckmann, D. S. Courson, A. Rybarska, C Hoege, J. Gharakhani, F. Jülicher, A. A. Hyman, Science 2009, 324 1729-1732.

[8] a) E. Dolgin, Nature 2018, 555, 300-302; b) S. Boeynaems, S. Alberti, N. L. Fawzi, T. Mittag, M. Polymenidou, F. Rousseau, J. Schymkowitz, J. Shorter, B. Wolozin, L. Van Den Bosch, Trends Cell Biol. 2018, 28 420-435; c) J. R. Vieregg, M. Lueckheide, A. B. Marciel, L. Leon, A. J. Bologna, J. R. Rivera, M. V. Tirrell, J. Am. Chem. Soc. 2018, 140 1632-1638.

[9] a) P. E. Wright, H. J. Dyson, Nat. Rev. Mol. Cell Bio. 2015, 16, 18-29; b) V. N. Uversky, Curr. Opin. Struct. Biol. 2017, 44, 18-30; c) V. N Uversky, I. M. Kuznetsova, K. K. Turoverov, B. Zaslavsky, FEBS Lett. 2015, 589, 15-22; d) M. Dzuricky, S. Roberts, A Chilkoti, Biochemistry 2018, 57, 2405-2414; e) B. S. Schuster, E. H. Reed, R. Parthasarathy, C. N. Jahnke, R. M. Caldwell, J. G. Bermudes, H. Ramage, M. C. Good D. A. Hammer, Nat. Commun. 2018, 9, 2985-2997.

[10] A. K. Dunker, J. D. Lawson, C. J. Brown, R. M. Williams, P. Romero, J. S. Oh, C. J. Oldfield, A. M. Campen, C. M. Ratliff, K. W. Hipps, J. Mol. Graphics Modell. 2001, 19, 26-59.

[11] a) S. R. MacEwan, I. Weitzhandler, I. Hoffmann, J. Genzer, M. Gradzielski, A. Chilkoti, Biomacromolecules 2017, 18, 599-609; b) S. C. Weber, C. P. Brangwynne, Cell 2012, 149, 1188-1191.

[12] a) J. R. Kramer, R. Petitdemange, L. Bataille, K. Bathany, A.-L. Wirotius, B. Garbay, T. J. Deming, E. Garanger, S. Lecommandoux, ACS Macro
Letters 2015, 4, 1283-1286; b) D. E. Meyer, A. Chilkoti, Biomacromolecules 2004, 5, 846-851; c) V. N. Uversky, Adv. Colloid Interface Sci. 2017, 239, 97-114.

[13] S. Roberts, M. Dzuricky, A. Chilkoti, FEBS Lett. 2015, 589, 2477-2486.

[14] a) J. R. Simon, N. J. Carroll, M. Rubinstein, A. Chilkoti, G. P. López, Nat. Chem. 2017, 9, 509-515; b) J. R. Simon, S. A. Eghtesadi, M. Dzuricky, L You, A. Chilkoti, Mol. Cell 2019, 75, 66-75; c) S. A. Costa, J. R. Simon, M. Amiram, L. Tang, S. Zauscher, E. M. Brustad, F. J. Isaacs, A. Chilkoti, Adv. Mater. 2018, 30, 1704878-1704887.

[15] R. J. Ellis, Trends Biochem. Sci 2001, 26, 597-604.

[16] a) C. D. Keating, Acc. Chem. Res. 2012, 45, 2114-2124; b) A. G. Teixeira, R. Agarwal, K. R. Ko, J. Grant-Burt, B. M. Leung, J. P. Frampton, Adv. Healthc. Mater. 2018, 7, 1701036-1701055; c) Y. Chao, H. C. Shum, Chem. Soc. Rev. 2020, 49, 114-142.

[17] P. A. Albertsson, Partition of cell particles and macromolecules, Wiley$\mathrm{VCH}, 1986$.

[18] M. R. Helfrich, L. K. Mangeney-Slavin, M. S. Long, K. Y. Djoko, C. D. Keating, J. Am. Chem. Soc. 2002, 124, 13374-13375.

[19] R. Petitdemange, E. Garanger, L. Bataille, K. Bathany, B. Garbay, T. J. Deming, S. Lecommandoux, Bioconjugate Chem. 2017, 28, 1403-1412.

[20] D. E. Meyer, A. Chilkoti, Biomacromolecules 2002, 3, 357-367.

[21] a) D. E. Meyer, A. Chilkoti, Nat. Biotechnol. 1999, 17, 1112-1115; b) R Petitdemange, E. Garanger, L. Bataille, W. Dieryck, K. Bathany, B. Garbay, T. J. Deming, S. Lecommandoux, Biomacromolecules 2017, $18,544-550$

[22] a) N. Nakatani, H. Sakuta, M. Hayashi, S. Tanaka, K. Takiguchi, K Tsumoto, K. Yoshikawa, ChemBioChem 2018, 19, 1370-1374; b) L. Breydo, L. M. Mikheeva, P. P. Madeira, B. Y. Zaslavsky, V. N. Uversky, Mol. Biosyst. 2013, 9, 3068-3079.

[23] S. An, R. Kumar, E. D. Sheets, S. J. Benkovic, Science 2008, 320, 103 106.

[24] E. Gomes, J. Shorter, J. Biol. Chem. 2019, 294, 7115-7127.

[25] a) S. D. Hann, T. H. R. Niepa, K. J. Stebe, D. Lee, Langmuir 2016, 8 25603-25611; b) D. C Dewey, C. A Strulson, D. N. Cacace, P. C. Bevilacqua, C. D. Keating, Nat. Commun. 2014, 5, 4670-4679.

[26] J. B. Sumner, N. Gralën, I.-B. Eriksson-Quensel, Science 1938, 87 395-396.

[27] L. Elling, M.-R. Kula, E. Hadas, E. Katchalski-Katzir, Anal. Biochem. 1991
192 


\section{Entry for the Table of Contents}

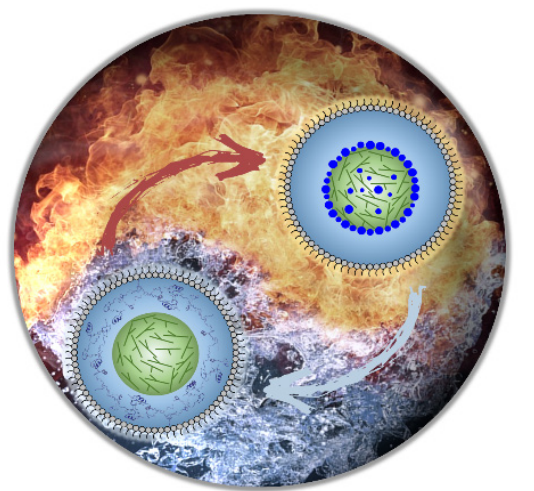

Intrinsically disordered proteins (IDPs) within cellular milieu can phase separate into membrane-less organelles. The phaseseparated feature of IDPs in intracellular environment has inspired the study of spatial distribution and dynamic coacervation of a thermo-responsive elastin-like polypeptide (ELP) as a model of artificial IDP inside synthetic cell-like chassis. 